\title{
Editorial
}

\section{Molecular Tumor Board}

\section{Nirmal Lamichhane}

\section{B P Koirala memorial Cancer hospital, Bharatpur, Chitwan}

A tumor board is a meeting made up of specialized doctors and other health care providers who regularly gather to discuss cancer cases that are unusual and/or challenging. The goal is to decide on the best possible treatment plan for a patient as a group. This has become a very systematic method in cancer care and is useful in picking up all the available information about the patients, inputs of all concerned doctors and other health care personnels.

Now a days there is much information about the tumor biology in molecular level and there are more drugs targeting molecular mechanisms of the cancers. With this developments, there is a new type discussion for index cancer patient involving molecular scientists and clinicians to see what would be the best possible care.

Nine per cent of patients with metastatic cancer harbour genomic alterations that are recognised as biomarkers for optimal treatment selection in current standard of care. An additional 27\% carries genomic aberrations for which compelling clinical evidence exists supporting the use of these alterations as predictive biomarkers for drug response outside the registered indication. ${ }^{1}$ As novel molecular and genomic treatment indications are explored in rapid pace, the generation and correct interpretation of molecular tumour profiles are quickly becoming a necessity for offering optimal cancer treatment. The complexity and vast amounts of data generated through molecular profiling techniques, like next-generation sequencing, make expert review an absolute requirement in order to translate molecular profiles into clinical benefit for our patients. Leading cancer care providers are currently trying to address this by developing the so called 'molecular tumour boards' (MTBs), which comprise experts of various disciplines who help clinicians to interpret the molecular profiles of their patients. This is a challenging task and many uncertainties about the optimal implementation of these boards remain. Among different institutions, implementations can differ on various grounds: technically (eg, the used sequencing techniques, bioinformatics pipelines), composition wise (eg, which types of specialists are involved) or organizationally (eg, centralized vs localized). A multidisciplinary team comprising physicians, a molecular oncologist, clinical geneticist and molecular pathologist evaluates each individual case submission, taking clinical history of the patient and (technical) details of the tumour specimen into account. The latter included examination of the tumour fraction and variant allele frequency, which could help in identifying germline variants that may be clinically relevant.

At the moment, less of the physicians even in a tertiary cancer centre reported on the lack of confidence in their genomic knowledge. ${ }^{2}$ This clearly stresses the need for education of oncologists in interpreting genomics data. Such improvement in molecular knowledge can only be achieved if all hospitals providing oncological care have access to MTBs that interpret sequencing data. Facilitating entry to MTBs for all hospitals will be challenging, but is essential to create equality in precision oncology care and should therefore be highly prioritised during implementation of MTBs in a country or region. ${ }^{3}$ This exposes that, apart from expert interpretation of molecular profiles, interesting additional challenges remain in order to exploit the full potential of precision oncology. Approved drugs are often inaccessible to biomarker- positive patients due to the absence of relevant drugs beyond their labelled indication and insurance issues even in developed world. It is likely that future MTBs are increasingly going to be exposed to novel molecular profiling techniques. For example, high-dimensional characterisation of the immune infiltrate, or functional experimentation with patient-derived organoids and/or immune cells might guide personalised immunotherapy treatment in the years to come. ${ }^{4}$ Taken together, the development of MTBs is a highly needed but dynamic and 
challenging process in a rapidly evolving field. More sharing and discussing their implementation of an MTB with the oncological community, sharing experiences on the implementation of MTBs will undoubtedly accelerate the quality of care in this area. This will promote the precise and individualized medicine in field of Oncology. LMIC are not exception, care is needing to move along this main stream of oncological development.

\section{References:}

1. Van de Haar J, Hoes L, Voest E. Advancing molecular tumour boards: highly needed to maximise the impact of precision medicine. ESMO Open 2019;4:e000516. doi:10.1136/ esmoopen-2019-000516
2. Claudio Luchini, Rita T. Lawlor, Michele Milella, and Aldo Scarpa. Molecular Tumor Boards in Clinical Practice.Trends in Cancer, September 2020, Vol. 6, No. 9, 738-744.

3. D. L. van der Velden1, C. M. L. van Herpen2, H. W. M. van Laarhoven3, E. F. Smit4, H. J. M. Groen5,S. M. Willems. Molecular Tumor Boards: current practice and future needs. Annals of Oncology 28: 30703075, 2017. doi:10.1093/annonc/mdx528

4. Annelieke E. C. A. B. Willemsen, Sarah Krausz, Marjolijn J. L. Ligtenberg, Katrien Grünberg, Harry J. M. Groen, Emile E. Voest. Molecular tumour boards and molecular diagnostics for patients with cancer in the Netherlands: experiences, challenges, and aspirations. British Journal of Cancer (2019) 121:3436; https://doi.org/10.1038/s41416-019-0489-3. 